\title{
Digital Human Modeling
}

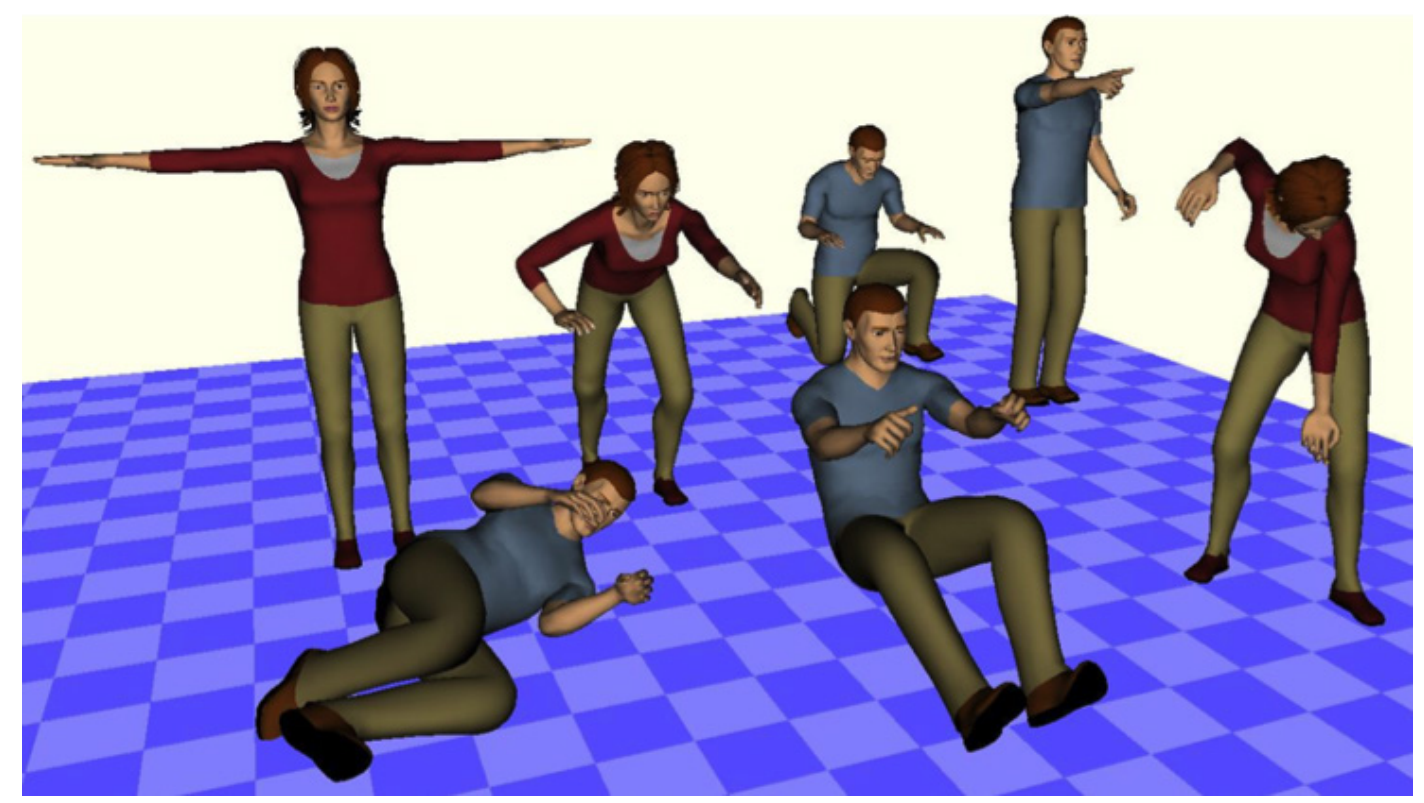

(c) 2016 Siemens Product Lifecycle Management Software Inc. Reprinted with permission.

\section{THIS CHAPTER PROVIDES:}

- A brief overview of the function of digital human modeling (DHM) and ergonomic simulations in the design of healthy workplaces.

- A walkthrough of ergonomics analysis functions commonly found in DHM software.

How to cite this book chapter:

Berlin, C and Adams C 2017 Production Ergonomics: Designing Work Systems to Support Optimal Human Performance. Pp. 161-174. London: Ubiquity Press. DOI: https://doi.org/10.5334/bbe.i. License: CC-BY 4.0 


\section{WHY DO I NEED TO KNOW THIS AS AN ENGINEER?}

Certain industrial sectors have strategically reached such a level of maturity regarding ergonomics that they have the means and equipment to simulate ergonomics in a computer, using a digital human model (called a manikin). Today, there are a number of commercially available 3D CAD software packages suited to ergonomic simulation, with built-in analysis functionalities that help an analyst to determine whether a new product or workplace design carries an ergonomic risk. Digital human modelling also offers the possibility to design and test new workplaces and products on a variety of virtual humans of different shapes and sizes. Knowing how to use these tools can save you time and money, allow you to compare several alternatives, and offer you better possibilities to design for a population of users, who might not be available in real life for testing.

\section{WHICH ROLES BENEFIT FROM THIS KNOWLEDGE?}

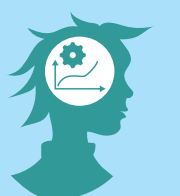

The system performance improver and work environment/safety specialist are both able to use ergonomics simulation as a way to make a business case for workplace improvements. Modelling the ergonomic risks and the impact of a design change is a fast, cheap, non-intrusive way to explain to other stakeholders what the immediate impacts of a change project would be (for example, introducing lifting equipment, changing a working height, etc.) without making changes that may disturb on-going production. The visualizations can also be used to communicate with suppliers of equipment or with operators about different risks and ways to address them.

The purchaser (and the system performance improver) can benefit from discussing a simulation of a proposed ergonomics intervention as part of the process of deciding whether the proposal is possible to fit in the available space, if it can successfully apply to all of the targeted workforce, and if the investment in e.g. new workspaces or equipment is likely to lessen the identified problematic loadings.

\subsection{Ergonomic simulation}

Simulations are used in many different sectors to test solutions before they are fully implemented and the discipline of ergonomics is no exception. Digital human modeling (DHM) is a term that designates a software tool that enables digital models of humans to interact with virtual workplaces or products in a digital CAD environment. The workplace can be built up in CAD and a number of tests done to determine its ergonomic suitability by importing a digital human, thus providing a visual representation of the workplace design in use. The size measurements of the digital human model are based on anthropometric databases, enabling a number of different models of different percentiles 
to be used in the same virtual workplace. There are a number of benefits provided by DHM for the production engineer:

- Easy to adopt a proactive design approach

- Enables numerous alternative solutions to be compared

- It is not always possible to access the real environment

- Easy to test a range of different measurements across different genders and nationalities

- Visualises the proposed work design layout and its effects on physical ergonomics, enabling meaningful discussions between designers, ergonomists and leadership

- Training aid

Given the high costs and large amount of space required to build full-scale models during the design and development stage, it can be difficult and costly to identify work tasks that may involve awkward postures or potential damaging body positions early on. Especially in the production environment where meaningful tests can only be conducted when the assembly line is shut down. Through the use of ergonomic simulations it is possible to make informed design decisions early on. Rather than only realizing there is an issue once the system has been implemented and injuries have started to occur, adopting a proactive approach and thoroughly testing and analysing design options early on through simulation can reduce injury risks and save time and money later in the implementation process.

As we have discussed throughout this book, production environments need to suit a diverse range of people with different sizes and strengths. Finding such a diverse range of people with enough time to conduct workplace testing can be difficult. However, with access to numerous databases and measurement sets, it is possible to import a range of different sized human models, of different genders and nationalities with varying percentiles using DHM. Care should be taken when using preloaded anthropometric databases, to ensure that the data is a true representation of the desired population. In addition to these testing functions, DHM can also be used as a training aid, so long before the workplace has been completed and implemented, operators can visually see what their tasks will look like.

A number of ergonomic simulation packages have been developed the last two decades, some of which are research projects and some of which are commercially available. Some noteworthy examples are:

- Jack (Siemens, 2016)

- RAMSIS (Human Solutions 2010; Bubb et al., 2006)

- SAMMIE (Marshall et al., 2010)

- DELMIA Ergonomics Specialist (Dassault Systèmes, 2016a); its well-known predecessor was DELMIA V5 Human (Dassault Systèmes, 2016b)

- Anybody (Anybody Technology, 2016)

- SANTOS (a "Virtual Soldier"; ESI Group, 2016; Abdel-Malek et al., 2007)

- IMMA (Hanson et al., 2012; 2014)

While the functionality and usability vary across the board, ultimately they all provide a method to test scenarios in a virtual environment using digital human models. Given that Siemens's software, Jack, is a well-known digital ergonomics evaluation tool that is easily researched online - and is 
offered with a free 30-day trial version - the majority of the terms used in this chapter will be exemplified with that software. However, many of the functions can typically be found within other programs (albeit with a different name).

\subsection{Computer manikins}

The human models used in ergonomic simulations are known as computer manikins and are a geometrical models of the human body that obey a set of biomechanical rules, with similar functional behaviour and capabilities as a real human. Given the complexity of the human body, with high levels of variation between individuals, it is impossible to create a truly accurate representation, so most manikins appear somewhat robotic in their movements. The computer manikins used in Jack are made up of 70 segments, 69 joints and 135 degrees of freedom. The manikins can be viewed as skeletons or as human representations as shown in Figure 9.1. Both female and male human representations exist within Jack software, Figure 9.2. Within the software it is possible to select a multitude of different sized models representing different percentile groups, Figure 9.3.

\subsection{Manipulation of manikins}

It is possible to move the manikins around to position them in certain ways, so that human postures and movements under certain task and environmental conditions can be simulated. In Jack this can be done in four different ways:

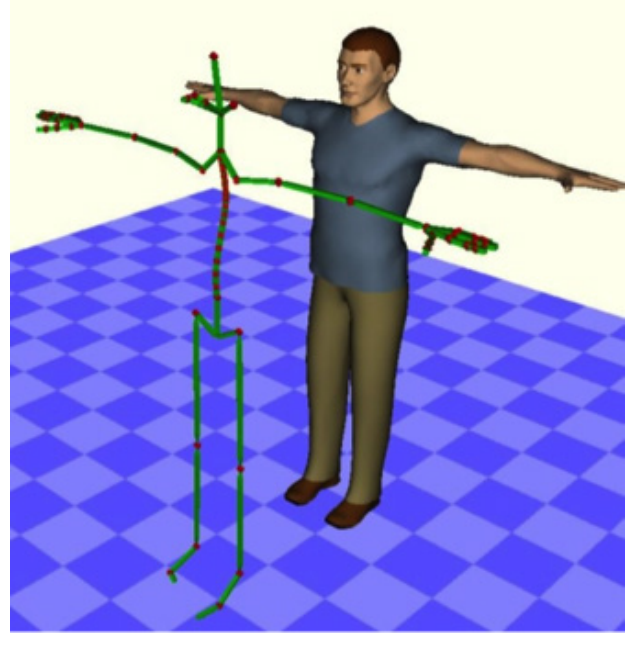

Figure 9.1: Computer manikin (skeleton and render) (Siemens, 2014).

(c) 2016 Siemens Product Lifecycle Management Software Inc. Reprinted with permission.

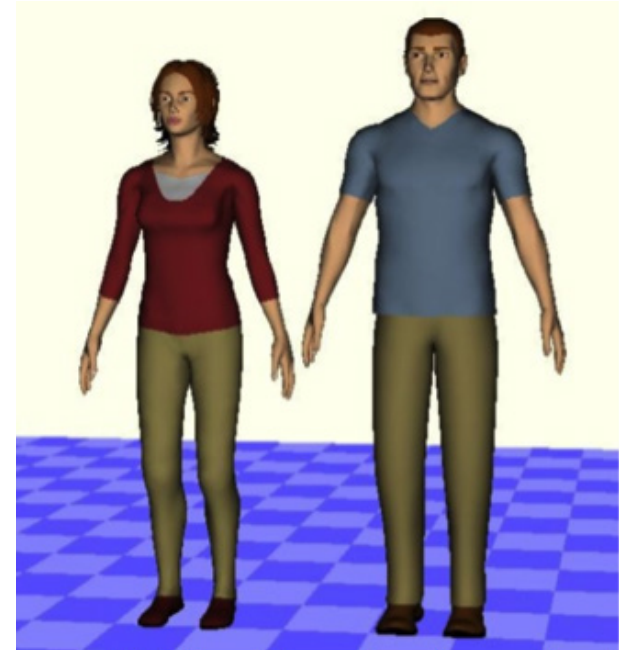

Figure 9.2: Male and female digital human models (Siemens, 2014).

(C) 2016 Siemens Product Lifecycle Management Software Inc. Reprinted with permission. 


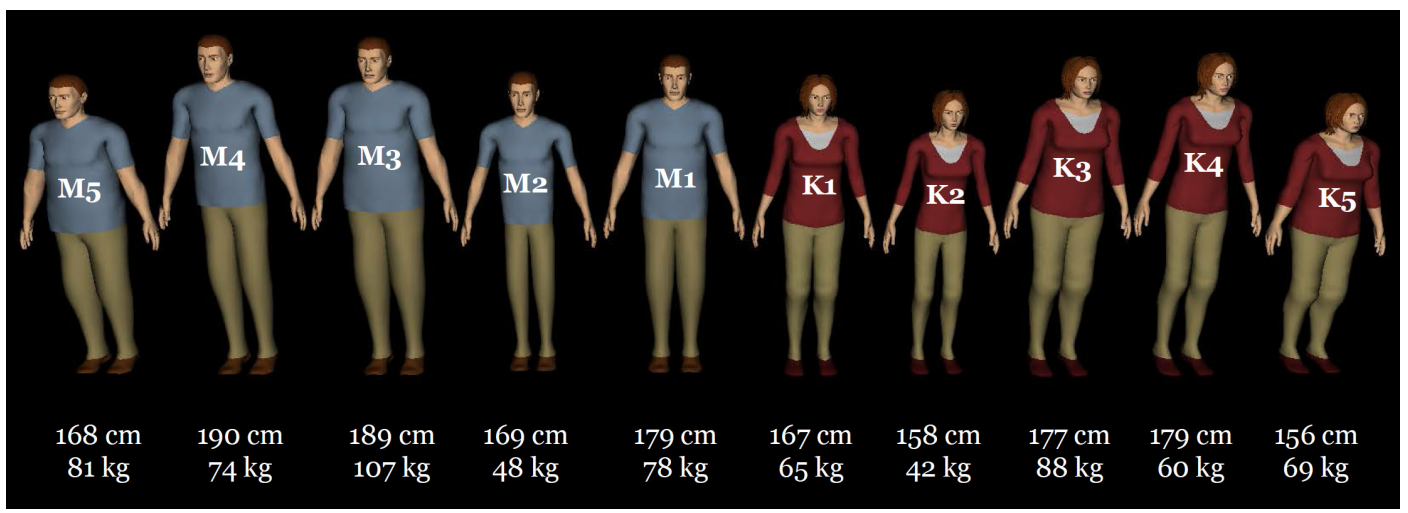

Figure 9.3: Digital human models with different percentile measurements (Brolin, 2013). (C) 2016 Siemens Product Lifecycle Management Software Inc. Reprinted with permission.

\section{Manipulating individual joints}

- Using inverse kinematics

- Using pre-recorded data

- Using the Posture Wizard

- Manipulating individual joints

The simplest way to move and position manikins is to select the individual joint and drag it in the desired distance in the $\mathrm{x}, \mathrm{y}$ or $\mathrm{z}$ axis (Figure 9.4). Ranges have been set within the software so theoretically the limbs can't be positioned outside the capabilities of the human body. However this is the most laborious method and achieving specified body postures can be very difficult.

\section{Inverse kinematics}

This is a mathematical method for controlling the movement of joints and position of the human body. While kinematics concerns calculating the position in space of the end of a linked structure, based all the different joint angles, inverse kinematics does the opposite. So the end point of the structure is known, e.g. the right thumb should be touching a button while the shoulder remains fixed, so the software will calculate what angle the joints should be at to allow this. However, given that in reality there is a range of possible solutions, in some instances the simulation might generate non-humanly possible movements based on the mathematical algorithm. Since this method can have a degree of error, combining it with manipulating individual joints can provide an accurate simulation.

\section{Pre-recorded data}

A number of common postures such as sitting and driving postures are stored within the program itself, so the user simply has to select the desired posture from a list and the manikin will automati- 


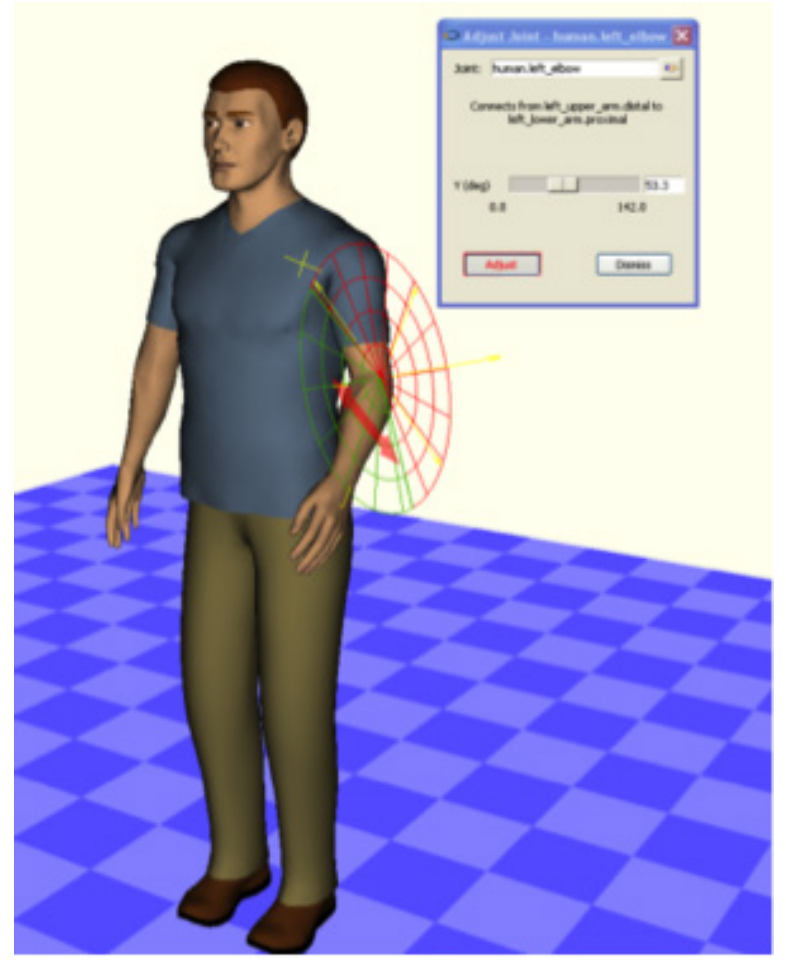

Figure 9.4: Manipulating joints (Siemens, 2014).

(C) 2016 Siemens Product Lifecycle Management Software Inc. Reprinted with permission.

cally adopt this position. The library of pre-recorded data is fairly extensive, with a range of different postures as shown in Figure 9.5; however, there will be some scenarios when the human model position needs to be set manually.

\section{Posture Wizard}

In this method, users set certain rules and constraints and the software creates postures accordingly. For example, in the case where an operator is leaning into a car exterior to attach the gear stick during assembly, constraints can be set regarding where both the feet and arms should be positioned so the operator can keep in balance without touching the shell. This method is considered faster and less fidgety than directly manipulating each individual joint of the manikin.

\subsection{Analysis tools}

In addition to manikin manipulation there are a number of other analysis tools within ergonomic simulation software that can aid in detecting risk areas early on. It is not possible or reasonable to 


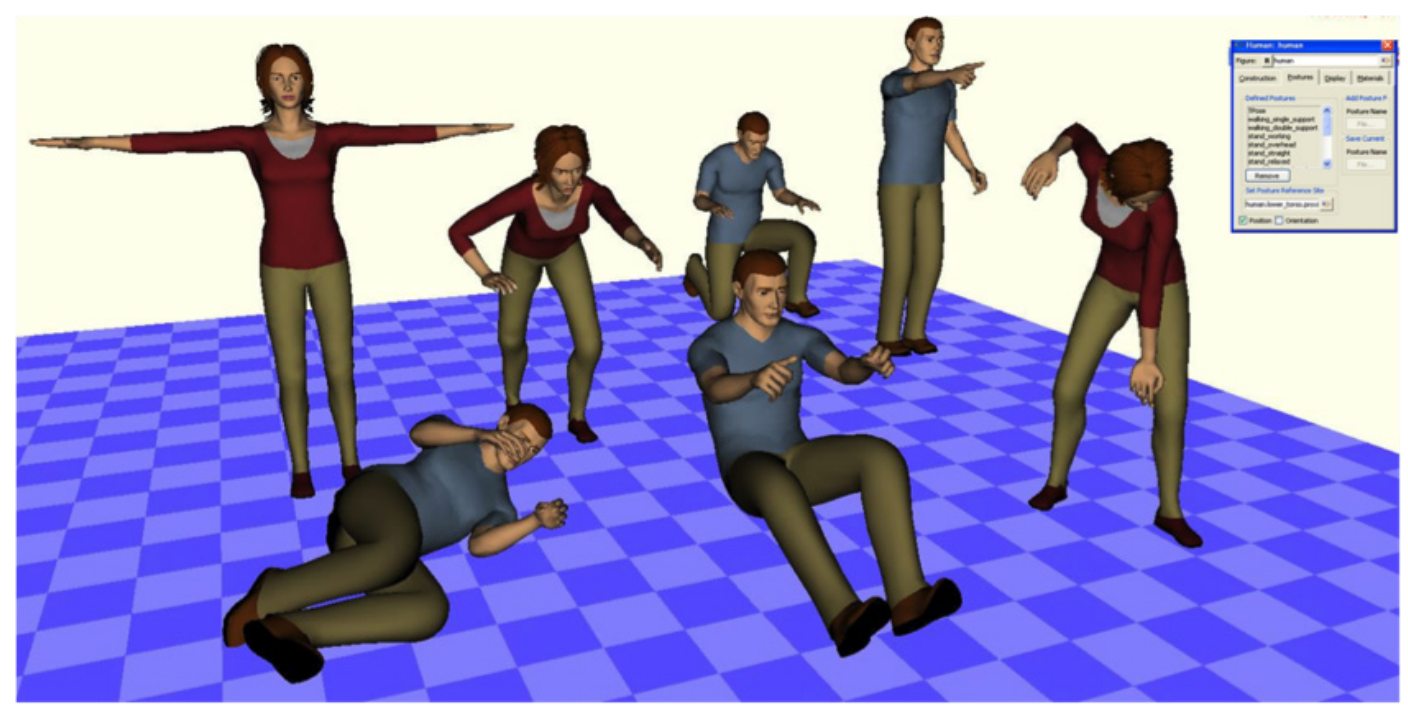

Figure 9.5: Pre-recorded postures in Jack (Siemens, 2014).

(c) 2016 Siemens Product Lifecycle Management Software Inc. Reprinted with permission.

cover all the features of ergonomic simulation tools in this chapter, so instead a brief overview of key features will be provided, to enable production engineers to grasp the bigger picture and better understand the possibilities that exist within DHM for carrying out ergonomic tests during the design phase. The following analysis tools will briefly be discussed:

- animation

- lower back analysis

- static strength prediction program

- comfort analysis

- RULA

- $\mathrm{NIOSH}$

- field of View

- space and reach

(However, it is important to note that this is not an exhaustive list.)

\section{Animation}

This feature allows users to make short animations, showing the human model carrying out the work tasks, enabling potential hazards to be identified. This can either be done as a key frame animation, where the user sets up the manikin posture for each phase and the software connects the phases, creating movements and the animation. Alternatively, motion capture tools (similar to those used in animated movies) can be used. In this case a real human conducts the task while wearing a special suit and data is collected to form an algorithm enabling a digital model and animation to be made. 


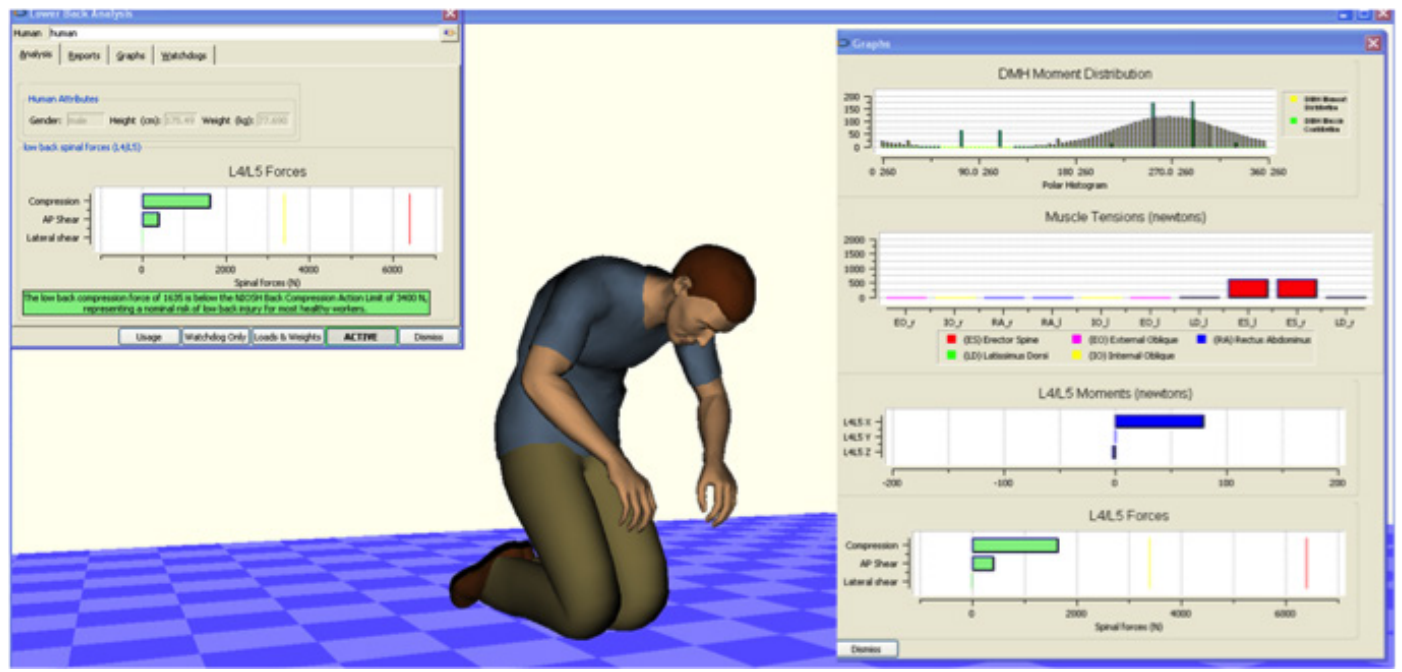

Figure 9.6: Lower back analysis tool in Jack (Siemens, 2014).

(c) 2016 Siemens Product Lifecycle Management Software Inc. Reprinted with permission.

\section{Lower back analysis}

This application forms a biomechanical model of the upper body of the manikin so that the static forces and torque present in the lumbar spine can be quantified. By comparing the simulated value with stored data the software can indicate whether the position is a risk or not. By having a value for the forces present in the lower back it is possible to compare alternative solutions and identify which presents the lowest level of risk. Figure 9.6 shows the output information provided by the software highlighting areas of high loading and which lumbers are experiencing the highest load.

\section{Static Strength Prediction Program (SSPP)}

This application uses biomechanical research to predict whether or not specified tasks are suitable for a certain population. This is particularly relevant for the production industry with a diverse working population of varying strength.

\section{Comfort analysis}

Another feature within DHM is comfort analysis. Given that quantifying something relatively subjective such as comfort is very dependent on posture and the environment, care should be taken when using this tool. A postural comfort metric had been established through a series of observations and empirical surveys. Responses from participants concerning comfort where collected while they were certain postures while carrying out specific tasks. These responses were then combined with joint angles measurement to create the postural comfort metric. 


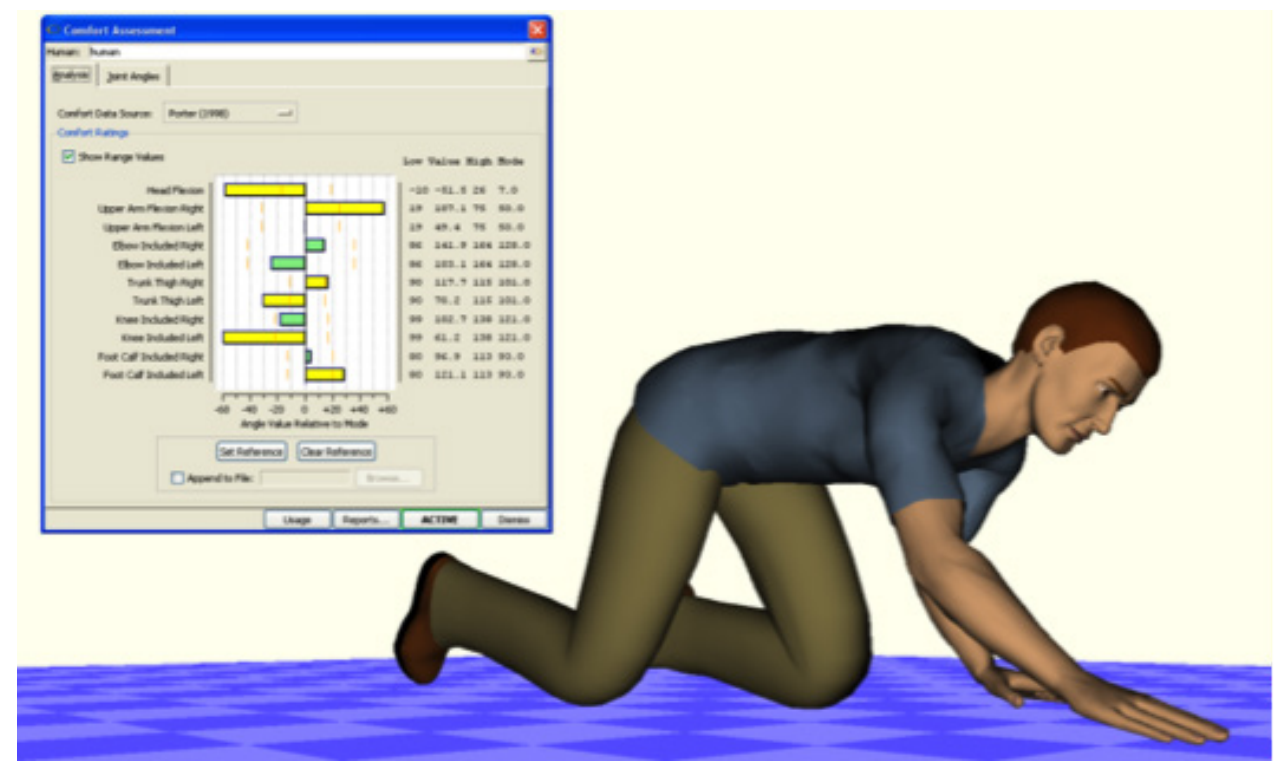

Figure 9.7: Comfort analysis tool in Jack (Siemens, 2014).

(c) 2016 Siemens Product Lifecycle Management Software Inc. Reprinted with permission.

By comparing these stored values with the simulated human model it is possible for the software to allocate each body section with a comfort-based score. The output of this analysis tool is shown in Figure 9.7, using colour coding to highlight instances and body parts that are regarded as uncomfortable.

\section{RULA}

DHM software also incorporates a number of ergonomic methods, some of which have been introduced in Chapter 6. For example, the built-in RULA tool can be used to identify the risk of triggering upper limb disorders in certain working postures. By inputting certain information, such as static loads along with the manikin posture the software can allocate a RULA score, indicating whether or not changes should be made to the work sequence (Figure 9.8). It is important to remember that observation-based methods, such as RULA, may be a bit "oversensitive" in DHM software, since joint angles are very exactly measured; this can sometimes result in dramatic changes in risk ratings based on very small changes in joint angles when they are near a specified angle threshold.

\section{$\mathrm{NIOSH}$}

Another ergonomic evaluation method that is built into the software is the NIOSH lifting equation. By providing input data about loads, frequency and posture of lifting tasks the tool uses the NIOSH lifting equation to determine whether the lifting load is acceptable. Providing the user with an analysis summary similar to that of the above RULA analysis. 


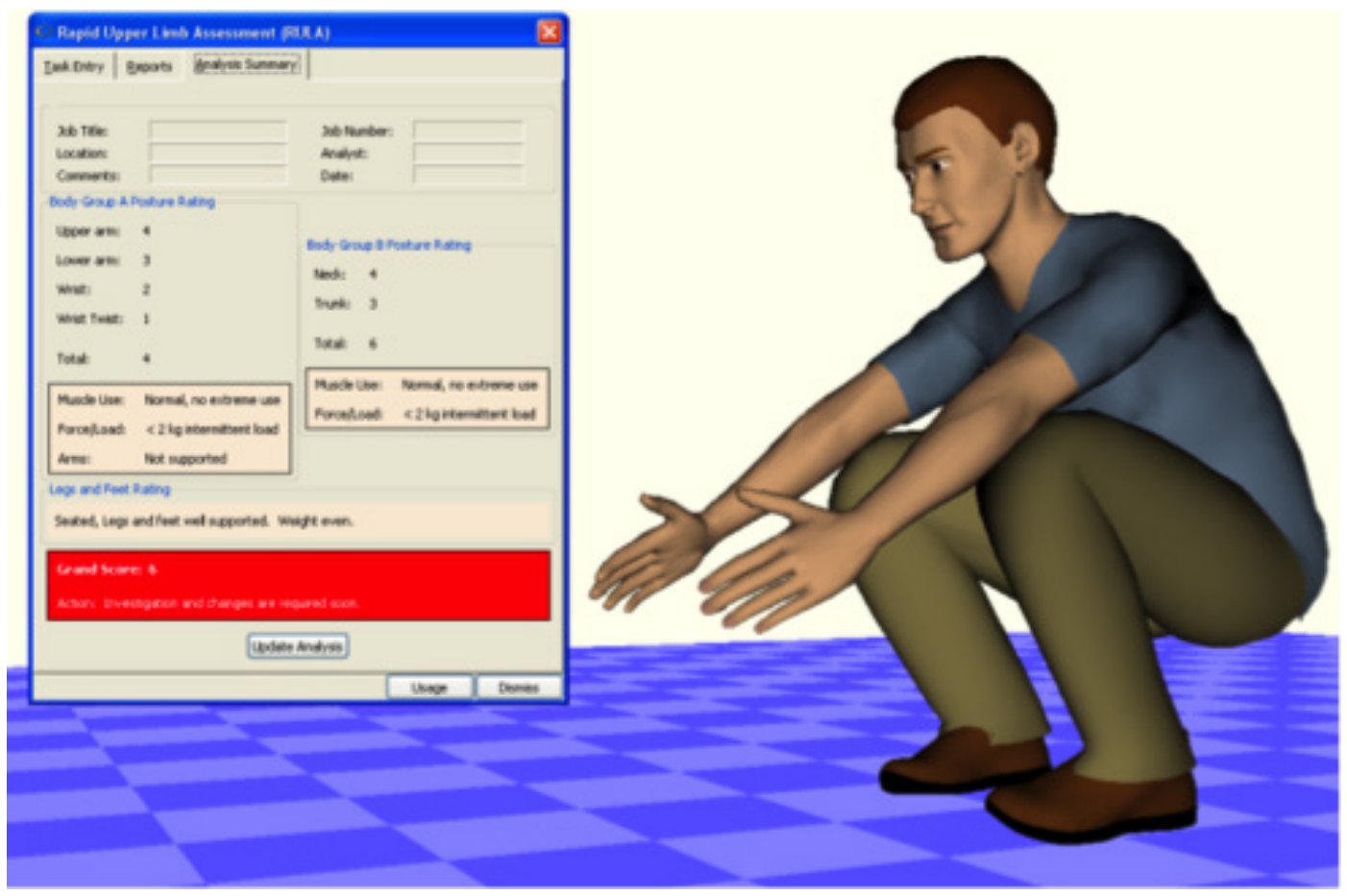

Figure 9.8: RULA tool in Jack (Siemens, 2014).

(C) 2016 Siemens Product Lifecycle Management Software Inc. Reprinted with permission.

\section{Field of view}

This tool provides information about the manikin's field of view, using coloured cones and boundary surfaces, a visual representation of the field of view for both eyes, the manikin's peripheral vision, their blind spot and what both the right and the left eye individually can see. Colour discrimination is also possible using this tool, so the tool gives information about areas where green, red, yellow or blue can be detected by the eye (Figure 9.9). This is particularly important when positioning warning signs. This tool makes it easy to check if screens or other necessary objects are directly in the manikin's field of vision, or if an alternate posture has to be adopted to see something.

\section{Space and reach}

The reach tool enables the areas of maximum and comfortable reach for each manikin to be easily visualized. This tool is particularly useful when ensuring the design is suitable for a diverse population and checking that all workers can components such as controls, pedals and levers. It can also be used to conduct accommodation studies to check that all the joints are provided with sufficient clearance zones. 


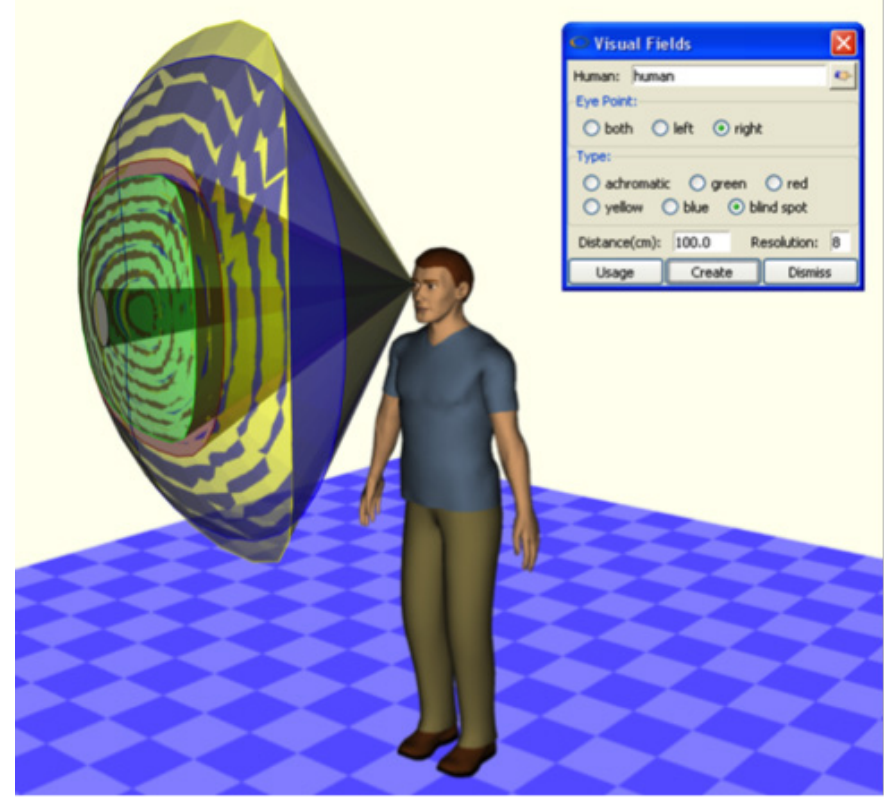

Figure 9.9: Field of view tool in Jack (Siemens, 2014).

(c) 2016 Siemens Product Lifecycle Management Software Inc. Reprinted with permission.

\section{Study questions}

\section{Warm-up:}

Q9.1) Name three reasons to use ergonomic simulation with DHMs before implementing a change to a workplace.

Q9.2) Name some common functionalities in DHM that can be useful as decision support in workplace design.

Q9.3) How are workforce populations represented in DHM?

\section{Look around you:}

Q9.4) The next time you encounter a 3D digital representation of a work environment or factory, determine if there is a human representation in it to indicate scale, and possibly usage scenarios (e.g. a story of what the operator needs to do and how that action proceeds). Does the human representation succeed in illustrating potential demands or problems in the workplace? Is it possible to assess the safety and suitability of the workplace to different worker sizes? 


\section{Connect this knowledge to an improvement project}

-When considering alternatives for a workplace design or re-design, DHM can be used to evaluate a 3D CAD model of the current or imagined environment to identify if particular tasks are supported or hindered by the design.

- The DHM allows the design to be tested on various manikin sizes (i.e. a manikin family) in order to determine whether the design is acceptable for the range of imaginable workers.

- The analysis methods and tools included in DHMs provide a quantification of the risk levels when different tasks and postures are carried out in the work environment.

\section{Connection to other topics in this book:}

- Basic biomechanics (Chapter 3) make up the basis for many computer manikins and their associated analysis capabilities. Knowing basic biomechanical principles can increase the understanding for (and critical evaluation of) DHM evaluation results.

- Knowing a bit about anthropometry (Chapter 4 ) and representative populations can aid the workplace designer in using DHM wisely as a design and testing tool.

- DHMs often include some ergonomics evaluation (Chapter 8) calculations as part of their analysis capabilities. Knowing how the most common ones are calculated based on observation data also increases the analyst's capacity for critical evaluation of the DHM results.

- In some companies, it may be well accepted to use DHM as a tool for involving stakeholder/ worker input (Chapter 6) by providing a visualization of the workplace, work tasks and improvement proposals.

\section{Summary}

- DHM tools enable a proactive approach to ergonomics by enabling virtual testing of designs early on.

- Using human manikins with various sizes and characteristics saves companies both money and time when considering various design alternatives.

- A range of different analysis methods can be conducted within the software to test the design's suitability, including injury risk, user comfort, reachability and line of sight.

- It is important to remember that observation-based methods, such as RULA, may be a bit "oversensitive" in DHM software due to the exact interpretation of the joint angles, meaning that some postures may be rated too severely, as the rating scales are discrete rather than incremental. 


\subsection{References}

Abdel-Malek, K., Yang, J., Kim, J. H., Marler, T., Beck, S., Swan, C., Frey-Law, L., Mathai, A., Murphy, C. \& Rahmatallah, S. (2007) Development of the virtual-human SantosTM. In International Conference on Digital Human Modeling (pp. 490-499). Springer Berlin Heidelberg.

Anybody Technology. (2016). Products \& Services. [Online]. Available from: http://www.anybodytech. com/ [Accessed 27 July 2016].

Brolin, E. (2013) Anthropometry. [Lecture] Chalmers University of Technology, 18th February 2013

Bubb, H., Engstler, F., Fritzsche, F., Mergl, C., Sabbah, O., Schaefer, P. \& Zacher, I. (2006). The development of RAMSIS in past and future as an example for the cooperation between industry and university. International Journal of Human Factors Modelling and Simulation, 1: 140-157.

Dassault Systèmes. (2016a). Ergonomics Specialist. [Online]. Available from: http://www.3ds.com/ products-services/delmia/capabilities/ergonomics/ergonomics-specialist-1/ [Accessed 27 July 2016].

Dassault Systèmes. (2016b). Human Workplace Design and Simulation. [Online]. Available from: http://www.3ds.com/fileadmin/PRODUCTS/DELMIA/PDF/Brochures/delmia-human-workplacedesign-simulation [Accessed 27 July 2016].

ESI Group. (2016). SANTOS [Online]. Available from: http://soldier.esi-group.com/Subpages/santos. html [Accessed 27 July 2016].

Hanson, L., Högberg, D., \& Söderholm, M. (2012). Digital test assembly of truck parts with the IMMA-tool-an illustrative case. Work, 41(Supplement 1): 2248-2252.

Hanson, L., Högberg, D., Carlson, J. S., Bohlin, R., Brolin, E., Delfs, N., Mårdberg, P., Gustafsson, S., Keyvani, A. \& Rhen, I.-M. (2014). 'IMMA-intelligently moving manikins in automotive applications' in Proceeding of ISHS 2014, Third International Summit on Human Simulation.

Human Solutions. (2010). RAMSIS (version 3.81.31). [Software] Kaiserslautern, Germany: Human Solutions GmbH.

Marshall, R., Case, K., Porter, M., Summerskill, S., Gyi, D., Davis, P. \& Sims, R. (2010). HADRIAN: a virtual approach to design for all. Journal of Engineering Design, 21: 253-273.

Siemens. (2014). Jack (Version 7-1) [Software] Siemens Product Lifecycle Management Software Inc. Siemens. (2016). Jack and Process Simulate Human. [Online]. Available from: https://www.plm.auto mation.siemens.com/en_us/products/tecnomatix/manufacturing-simulation/human-ergonomics/ jack.shtml

\section{Bibliography}

Siemens. (2014). Jack (Version 7-1) User Manual [Software] Siemens Product Lifecycle Management Software Inc. 
